\title{
Smart Agent Learning based Hotel Search System- Android Environment
}

\author{
Wayne Lawrence ${ }^{1}$, Suresh Sankaranarayanan ${ }^{2,3}$ \\ ${ }^{1}$ Mona Institute of Applied Sciences, University of WestIndies, Jamaica \\ wayne.lawrence.vip@gmail.com \\ ${ }^{2}$ Computing \& Information Systems, Institut Teknologi Brunei, Brunei \\ ${ }^{3}$ Department of Computing, University of WestIndies, Jamaica \\ pessuresh@hotmail.com
}

\begin{abstract}
The process of finding the finest hotel in central location is time consuming, information overload and overwhelming and in some cases poses a security risk to the client. Over time with competition in the market among travel agents and hotels, the process of hotel search and booking has improved with the advances in technology. Various web sites allow a user to select a destination from a pull-down list along with several categories to suit one's preference.. Some of the more advanced web sites allow for a search of the destination via a map for example hotelguidge.com and jamaica.hotels.hu. Recently good amount of work been carried in the use of Intelligent agents towards hotel search on J2ME based mobile handset which still has some weakness. The proposed system so developed uses smart software agents that overcomes the weakness in the previous system by collaborating among themselves and search Google map based on criteria selected by the user and return results to the client that is precise and best suit the user requirements. In addition, the agent possesses learning capability of searching the hotels too which is based on past search experience. The booking of hotel involving cryptography has not been incorporated in this research paper and been published elsewhere. This will be facilitated on Android 2.2-enabled mobile phone using JADE-LEAP Agent development kit.
\end{abstract}

Index Terms - Agents, JADE-LEAP, Android

\section{Introduction}

Hotel search and booking [1][2] is something which is done by everyone who wishes to travel and stay. This is normally carried out by travel agents. The process of finding the finest hotel in central location is time consuming, information overload and overwhelming and in some cases poses a security risk to the client. Over time with competition in the market among travel agents and hotels, the process of hotel search and booking has improved with the advances in technology. Various web sites allow a user to select a destination from a pull-down list along with several categories to suit one's preference. This with hotels putting up their own sites from which one would enter arrival date and departure dates to check availability and make a reservation. Some of the more advanced ones allow for a search of the destination via a map. Web sites such as hotelguide offer the user minimal input and then search Google map for the location requested or the user can find the location on the map and all hotels popup based on a star rating chosen by the user and booking is also done securely with Secure Socket Layer i.e. SSL [3]. The results are listed with the cost per hotel along with star rating. Another site jamaica.hotels.hu has a similar approach where the user has the choice of using the pull down or Google Maps for the destination and also criteria for the search such as a hotel with bed and breakfast. After selection of the desired hotel booking can be made from the site with Secure Sockets Layer (SSL) for security. Trip Advisor is one the most recognized popular hotel booking website sites that incorporates guest reviews along with other online travel agency sites and portals. Guest reviews add the credibility component that enhances the trust in your product. In all these hotel search systems, we see that there are lots of difficulties involved for users like time, speed, cost etc. To alleviate this issue, recently a lot of research [4][5] has being carried out on intelligent agent to replace the human agent to improve the speed and cost of search which however lacked the security component. Thus it is still predominantly a hassle and not 100 percent secure when it comes around to the actual booking of a hotel.

These systems would be discussed in detail in later section. So with all these in mind we here have developed [6] a system based on smart agents to search Google map for a hotel based on a guided list of criteria selected by the user. The search agent will make intelligent decision towards retrieving and sending results back to the mobile user similar to a human agent and also show the location of hotel on GMAP too. The system however even allows the agent to search hotels with limited search criteria by means of Agent learning concept i.e. based on past search experience of the agent. The booking of hotel involving Biometrics has not been presented in this paper and been published elsewhere [7]. The paper is organized in sections as follows. Section 2 provides details on Artificial intelligence in hotel search system followed by intelligent agent and biometric security. Section 3 gives 
details on the Intelligent Agent based hotel search and booking architecture followed by flowchart and algorithm. Section 4 gives the implementation details on JADE-LEAP and Android 2.2 with Google Maps API. Section 5 is conclusion and future work.

\section{Artificial Intelligence in Hotel Search}

Hotel booking systems (HBS) have been around for decades and over the years have become more interactive, providing clients of hotels worldwide to book a reservation without having to physically walk into the facility. The need for providing HBS that are intelligent in nature is therefore inevitable. In this section we explore some uses of AI by researchers in HBS with the aim of providing greater customer satisfaction, more efficient and reliable means of carrying out booking activities. Reference [8] focuses on definition of an information agent as "software programs that gather information from various heterogeneous and distributed sources on behalf of their human users to achieve their goals." Their solution was web-based but provided users with a single user interface that interconnects and traverses several other vendor websites and parallel processes the search request to achieve the goal. Reference [9] showed an implementation of a hotel recommendation system that was achieved through the use of an expert system which asked users a series of questions and based on the response would generate a recommended hotel for booking. Reference [2] in his work showed an implementation of an intelligent hotel reservation (IHR) that was accomplished using WAP enable mobile devices using XML to facilitate communication and MySQL as the database support.

Intelligent hotel booking, utilizing the Wireless Application Protocol (WAP), along with JAVA as the programming language and XML are perfect match for each. The developed prototype allowed the potential guest to make room reservations, obtain hotel information and by interacting with an automated helpdesk through a WAP supported mobile device they were able to garner more understanding. It has always been the habit of mankind to find solutions that are less labor intensive, but provides comparative or superior means of accomplishing given tasks. Developers of HSBs or hotel reservation systems are moving towards more intelligent solutions for clients. The need for this comes as they seek to satisfy clients with efficient and cost-effective ways in reserving a hotel for vacations or business ventures. Available literatures have proven that integrating artificial intelligence in HBS can satisfy the need for cost-effective, efficient and reliable hotel booking. Now with is in mind, we will look into some of Agent based Hotel booking system been developed for mobile device which was the motivation towards our research. But before that we will look into Agent Technologies.

\section{A. Agent Technologies}

Agent based technology falls within the field of Artificial Intelligence. An agent should be autonomous in nature, having the ability to control its own action. Agents in principle are self-reliant software programs [10]. It is critical that agents are intelligent in nature. This means they should possess the ability to sense the environment that they are in and adapt accordingly. Reference [11] discussed the flexible nature of agents. They are of the view that the agent should be adaptive, knowing how to adjust to its environment.

Whilst Agent technology is not relatively new to the study of AI, the need to have a clear understanding of its capabilities is important. Agents usually require several inputs before they can arrive at an action which is outlined in [12].

Another point to note about agent is their mobility. Agents should be able to move from environment to environment. The portability of agents is crucial to facilitate information exchange [13]. To effectively develop or program intelligent agents, several agent development kits have been developed. Agent toolkits help programmers of agent-base systems to have an environment in which they can develop, simulate, test or monitor agent's performances before actual deployment of the application. Reference [14] identified at least four categories of agent toolkits used today. They are general-purpose agent toolkits, multiagent toolkits, internet agent toolkit and mobile agent toolkits. Each of the preceding categories of agent toolkit has been used in agent-base solutions.

\section{B. Agent Learning}

Corporative multi-agent learning [15] approaches can be segmented into two major categories: Team learning approach and the concurrent learning. Concurrent learning utilizes multiple concurrent learning processes. Rather than learning behaviors for the entire team, concurrent learning methods normally employ a learner for each team member. This is done with the expectation that it reduces the joint space by projecting it into $N$ separate spaces. However, the presence of multiple concurrent learners makes the environment mobile. This in itself is a contravention of the assumptions behind most conventional machine learning techniques. This is the main reason why concurrent learning needs new or significantly modified forms of machine learning methods.

Team learning involves a single learner which discovers a set of behaviors for a team of agents instead of a single agent. Team learning can use regular singleagent machine learning procedures to accomplish its goals. Team learning can be separated into two type's namely homogeneous and heterogeneous team learning. Homogeneous learners would advance using single agent behavior which by extension is then used by every agent on the team. Heterogeneous team learners 
on the other hand, can develop based on the distinctive behavior for each agent. Heterogeneous learners are therefore expected to yield superior solutions from agent specialization. A middle ground is found by using hybrid learning.

\section{Agent based Mobile Hotel Search system}

There has been some work been done in the use of intelligent Agents in Hotel search on mobile handsets. Reference [4] explored this possibility in their research to develop an intelligent agent based mobile tour planner. Their work featured the implementation of the tour planner using JADE-LEAP [16][17] as the main platform for the system that interface with a mobile device to provide user with the ability to do searching for hotels by performing some minimal search criteria. The shortcoming of their work was that there was no means provided for users to immediately reserve a hotel of their choice. Also their search system gave no information about the feedback of the hotel. The intelligence provided was very minimal where search was done purely on price only. Based on the above work, Reference [5] developed the system by adding more intelligence to the search operation and implementing not only a booking agent, but a means for users to provide some feedback on their experience at a given hotel that they have stayed. This information is captured into a centralized area that will be used to provide some rating to users, to help them in decisionmaking.

In all these Agent based systems, there has been no GMAP facility included for searching the hotels and retrieving the results. Also these agents possess no learning capability i.e. past search experience to search the hotels. Finally, no security implemented towards booking the hotel by giving financial information. So we here in our smart agent based system have implemented GMAP facility and Agent learning capability towards hotel search and Biometrics towards booking the hotel has been published elsewhere[7]. Detail on our system been explained in forthcoming sections.

\section{Intelligent agent based Hotel Search and Booking System}

There has been quite amount of work carried out in the use of intelligent agents in hotel search systems which is been discussed before. So taking those into consideration, we here have improvised the Agent based system towards hotel search and booking i.e. SAL-HSB which would use the intelligence to search for hotels using the GMAP facility. In addition, the agent possesses intelligence to search hotels based on previous search experience of the agent which is Agent learning feature. Finally, the system possesses Biometric features to book hotel without needing to provide card information and details towards these are published elsewhere [7]. The Smart Agent Learning for Hotel Search and Booking System (SAL-HSB) [6] consist of the five agents shown in Fig. 1. The details of agents related to search been discussed here whereas agent related to booking like Booking Agent, Card Agent, Trusted Third Party and Security agent which forms a part of Hotel booking and cancellation are been published elsewhere [7].

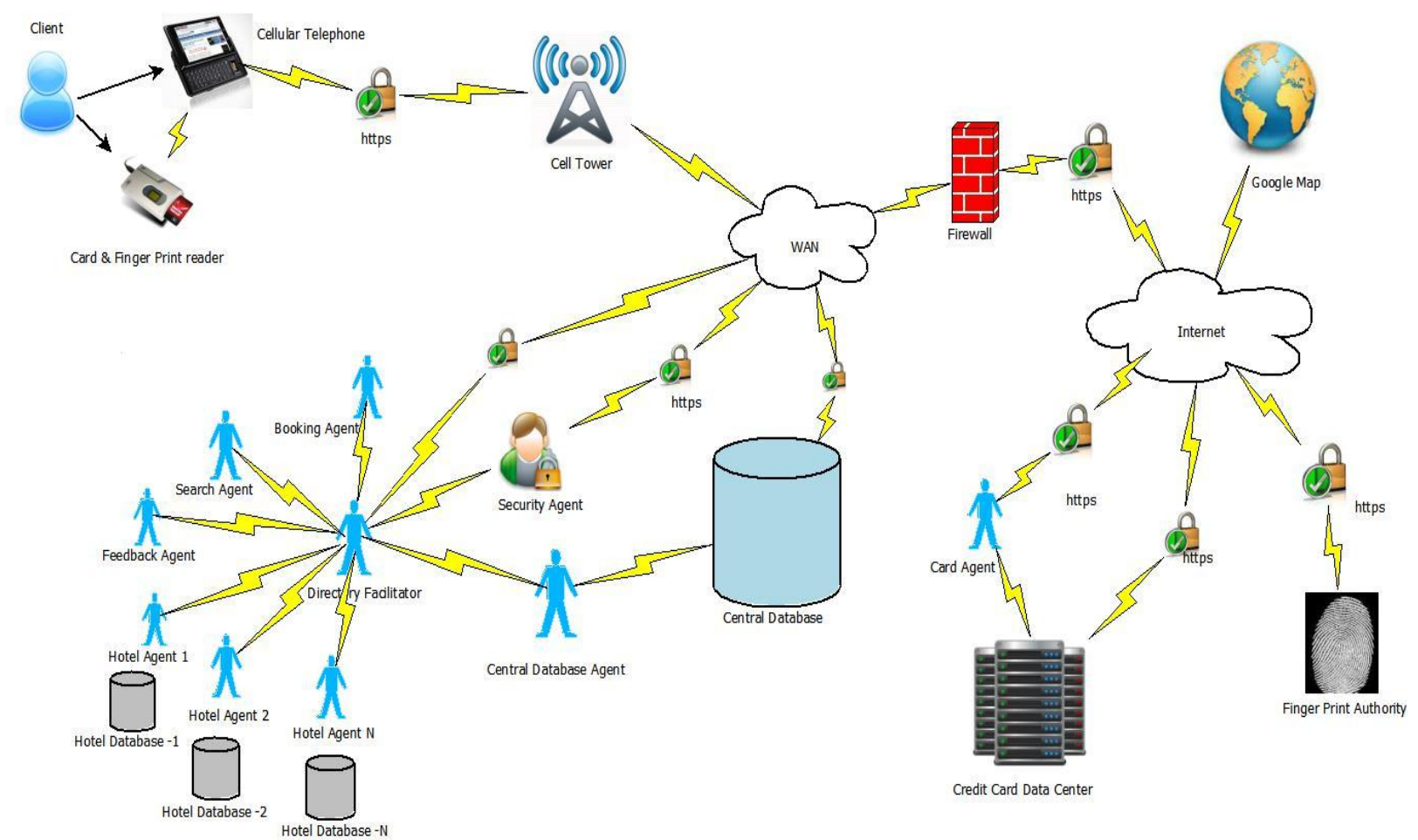

Fig 1 SAL-HSB System Architecture 


\section{A. Hotel Agent (HA)}

The hotel agent equips the central database agent with all the information regarding the hotel operation and services that is offered by the hotel. This is collected and stored by the central database agent for future use. Each hotel would host an agent that is registered with SAL-HSB application. Booking and cancellation information is also passed between the booking agent and the hotel agent to ensure that the hotel reservation system is updated and available for access by representatives of the hotel staff.

\section{B. Hotel Search Agent (HSA)}

This is the main agent of the application on which other features rely. The mobile device provides hosting for this agent.

Smart searches are performed based on user criterion and fuzzy preference rules and maybe adjusted dynamically to facilitate the most accurate and best suited results

$>$ Smart searches are executed with calculation of popularity index and results sorted in descending order automatically when user selects certain criteria.

> Hotel ratings are also incorporated with search results once user selects certain criteria.

$>$ Warning messages are also relayed for display to the end user once popularity index or rating falls below a prescribed level of acceptance.

\section{Central Database Agent (CDA)}

The Central Database Agent is one of the major players in the overall architecture that enables communication, accumulating and dispatching information which is situated on the central repository of the system. The main function of this agent is to capture and store information relating to the feedback feature implemented, collect information from all hotel agents about respective hotels which include data such as address, pricing and facilities, process query submitted by the search agent against data stored in the central database, querying the Directory Facilitator (DF) to know the registered hotel agents and executing popularity index calculations.

\section{Feedback Agent (FA)}

The feedback mechanism facilitates and simplifies the process to gather user ratings from those who were able to book a hotel and have experienced the services offered by the hotel. The areas of interest were infrastructure, environment, ambience, facilities, customer service, room service, security, disability access and price. The ratings for all areas are collected and then stored by the central database agent. This information is readily available for the feedback agent to query, scrutinize for time intervals of three months, six months and one year. The outcome of which is sent to the end user via the search agent for presentation of results. The feedback agent is hosted on the mobile device.

\section{E. Directory Facilitator (DF)}

The directory facilitator provides four methods for developers to interface with namely register, modify, deregister and search to aid in agent development and yellow page lookup of services for registered agents. All authorized agents are eligible for the services that are provided by the directory facilitator once registered. This includes a precise up-to-date list of all agents and services they provide. Agents can afterwards request the deregistration of a description at which time there is no longer an obligation on behalf of the directory facilitator to broker information on behalf of that agent. The agent is also able to request the directory facilitator to modify its agent description at any point in time for any reason. Agents can issue search requests to the directory facilitator to ascertain descriptions matching supplied search parameters.

\section{F. Mobile Device}

An android 2.2 smart phone would be required by the end user for interfacing with our SAL-HSB application supported by a mobile network with reliable internet access and data services in order to allow for uninterrupted communication with the agent platform and other services on the internet for example Google maps API. This can also be supplemented with the use of an android emulator that mimics the mobile device when being developed.

Having discussed the various agents in the SAL-HSB system developed, we will see in detail the Hotel Search process which is been presented in algorithmic fashion. The process of booking and cancellation are published elsewhere [7].

\section{Algorithms in SAL-HSB}

\section{A. Search Agent Algorithm}

* User enters hotel search criteria from a GUI on mobile device (Intelligent Agent), such as: Parish \& Location using GMAP, price, percentage markup, rating, facilities etc.

* Upon submission the search criteria is passed to the hotel search agent that starts the search process sending the request to the database agent to look up a list of suitable hotels matching the search criteria as follows:

If a hotel is available for lower price range with exact or closest matching of amenities in the same or different location or parish If a hotel is available for the price range specified with exact or closest matching of amenities in the same or different location or parish 
If a hotel is not available within the price range, it finds a hotel above the maximum price by say 5, 10 or $25 \%$ more than his/her maximum price with exact or closest matching of amenities in same or different location or parish

$>$ If no hotels available within the price range or so, it finds a hotel with the facilities for any price range in same or different location or parish

* The hotel search agent interacts with a database agent that in turn forwards the results to the search agent along with the rating and popularity information

* The search agent returns the result and displays it on the GUI of the mobile devices.

\section{B. Agent Learning Algorithm}

The algorithm used for Agent learning is same as above except here the user enters only the mandatory hotel search criteria from a GUI on mobile device such as: Parish \& Location using GMAP, price, percentage markup, rating only. Facilities are left as blank as it is assumed that sometimes users find it cumbersome to select all facilities mentioned in the system. So instead of just taking default value and searching the hotels as in previous system [5], We here use Agent learning wherein the hotel search agent interacts with a database agent and retrieves the hotels with maximum popularity i.e. ones which is most preferred by the users based on past search experience and forwards the results along with the facilities, rating and popularity on the GUI of the mobile device. The same fuzzy preferences are used for searching except facilities are left as blank.

\section{Implementation using JADE-LEAP}

SAL-HSB [6] Implementation was done using android SDK 11.0 and java development kit 1.6.0_25 (Jdk1.6.0_25) as the runtime environment using Eclipse Indigo release build id: 20110615-0604 with Android plug-in enabled as the integrated development environment IDE. This is an open source IDE which is freely available for download with many features for java developers. JADE 4.0.1 was used for the agent platform to develop, control, manage and host all agents. Jade Android 1.0 enabled JADE-LEAP agents on the mobile device. Android platform 2.2 [18], API level 8 with Google APIs was used to build the application with Google maps capabilities. Our data needs were all met by Microsoft SQL Server 2008 [19] developer edition. This was used to create all databases needed for our centralized hotels. The system i.e. SALHSB is created using Android 2.2 [19] with Google API 8 and Java Agent Development Toolkit (JADE) with Lightweight Extensible Authentication Protocol (LEAP) [16][17].The list of agents in the JADE environment is shown in Fig. 2 The Central Database Agent resides in the main container and is labeled HSBDB Agent. All other agents reside in separate split container and are identified by their names

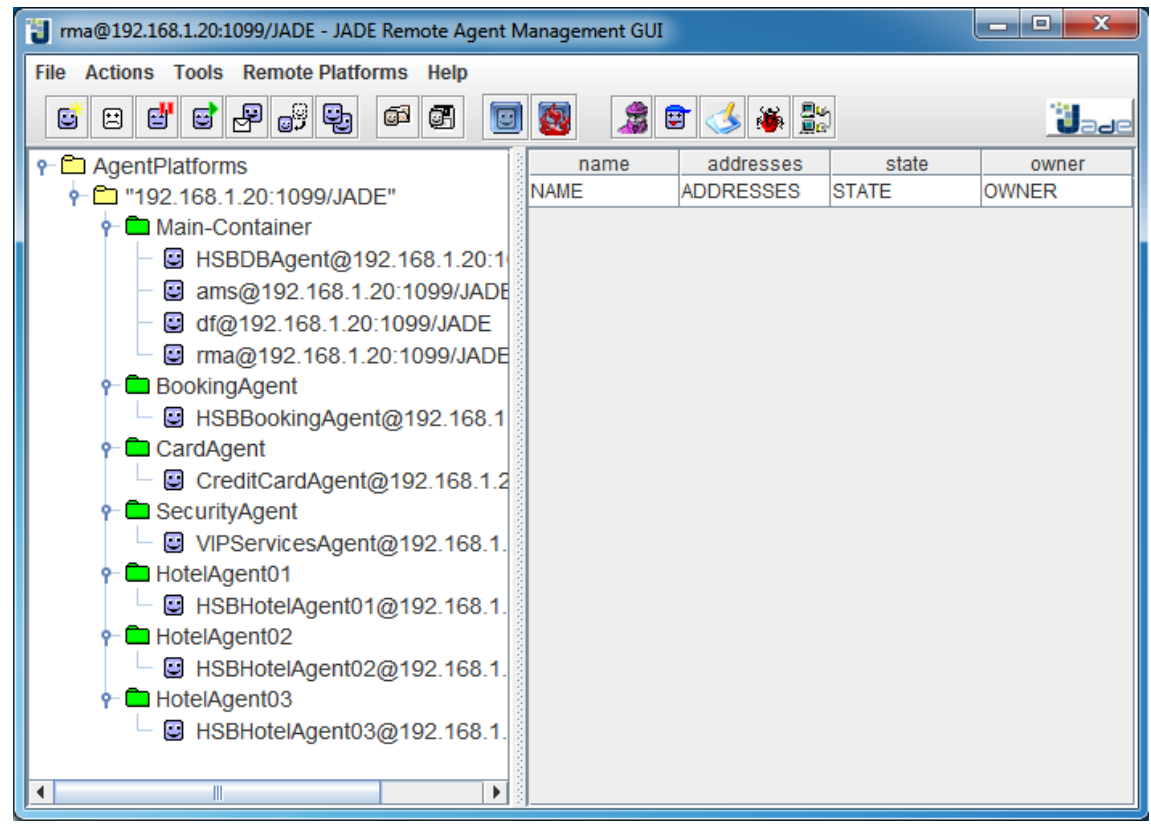

Fig 2 Agents in JADE Container

\section{A. Hotel Search}

The hotel searches are performed using an Android enabled mobile device with access to the internet and fairly good connective to the cellular network. Entry into the search menu will initiate the Hotel Search
Agent into operation. Among the options the user has to configure for his or her search are category, star rating, price markup, price range, room view, breakfast, food, facilities and activities. A detailed description is given below: 
- Location - This mandatory and user is assisted by Google Maps with this task

- Category - All Hotels, Hotels (rated 1 star to 5 star) and Villas

- Star Rating - This is a rating standard used by the industry $(1-5)$

- Price Markup - 5 to 30 percent with increments of 5

- Price Range - Low of $\$ 100.00$ (US) to high of $\$ 1100.00$ (US)

- Room View - Garden, Ocean, Mountains and City view

- Breakfast - yes or no

- Food included - Yes or No

- Feedback - Periods of 3 months, 6 months and one year are available for selection

- Facilities - Restaurants, Internet, Bar, and parking etc.

- Activities - Tennis, Golf, Scuba diving and casino etc.

Many of these options are optional and default values are used in case where option is not chosen by the user. When the user is satisfied that the required or desired interests are met or configured on the criteria search interface, the search button is clicked to initiate the search operation which is primarily driven by the Hotel Search Agent (HSA).We will present few scenarios along with screen shots to substantiate the intelligence so developed for our system.

Let us consider a scenario where user will select a destination from the Google map interface by navigating around using the zoom controls and mouse to select a desired location/region as shown in Fig. 3. The user here selects the location Negril aided by Google maps depicted in Fig. 4. After selecting the location, user selects user criteria such as Price less than 200.00, Breakfast and Food included with feedback of 3 months. The other facilities and activities are left as blank where the system would take default value as shown in Fig. 5. Now based on the input, the hotel search agent from the mobile would contact with the central database agent which possess intelligence to find the hotels for price less than 200.00 in location Negril with Breakfast and food included. The central database return the list of hotels for price less than 200.00 in the location Negril with rating and popularity information where popularity shows how popular the hotel is in the location as shown in Fig. 6 based on the number of persons who have stayed. From the results as shown in Fig. 6, the hotels are listed with the rating of 4 and popularity above $60 \%$ which is excellent. Fig. 7 shows the location of Hotels in Google Map.

Rating is calculated over scale of 1 to 5 based on factors like Infrastructure, Environment, and Ambience etc. We define here the popularity index as follows:

Popularity index $=$ (number of persons stayed/number of persons booked)*100.
Also popularity index is graded as follows:

- $60 \%$ and above : Excellent

- $30 \%$ and above to less than 60\%: Good

- $15 \%$ and above to less than 30\%: Average

- $0 \%$ and above less than 15\%: Poor

Let us consider a scenario where user selects the location Kingston aided by Google map as before. After selecting the location, user selects criteria such as Price less than 300.00 with price markup of $30 \%$ and feedback of 6 months. The other facilities and activities are left as blank as before where the system would take default value. Now based on the input, the hotel search agent from the mobile would contact with the central database agent which possess intelligence to find the hotels for price less than 300.00 in location Kingston with price markup of $30 \%$. The central database return the list of hotels for price less than 300.00 and also above 300.00 with markup of $30 \%$ i.e. 390.00 in the location Kingston with rating and popularity information as shown in Fig. 8. From the results as shown in Fig. 8, Spanish Court hotel listed got rating of 3 and popularity above $60 \%$ which is excellent. The other hotels got poor rating and popularity. Fig. 9 shows the location of Hotel in Google Map.

Let us consider another scenario where user selects the location Kingston aided by Google map. After selecting the location, user selects criteria such as Price less than 300.00 with some facilities like Room Service, Laundry facilities, Bar, Internet Access Swimming pool selected, Activities like Tennis unlike in other scenarios and feedback of 3 months. Now based on the input, the hotel search agent from the mobile would contact with the central database agent which possess intelligence to find the hotels for price less than 300.00 in location Kingston with facilities selected. The central database agent possess intelligence to return the list of hotels for price less than 300.00 in the location Kingston with rating and popularity information as shown in Fig. 10. Fig. 10 shows the hotel with available facilities with rating of $4 \& 3$ and popularity of above $30 \%$ which is good. The other hotel got $0 \%$ popularity and rating. Fig. 11 show that the facilities for Strawberry Hill Hotel and Spa does not have tennis or room service facilities however based on other matched facilities (bar, swimming pool and internet access) offered it was selected by our agent for the result list. Fig. 12 shows that based on the criteria The Jamaica Pegasus Hotel currently offers all facilities that were asked namely laundry facilities, tennis, room service, restaurant, bar, internet access and a swimming pool. Therefore our agent added this hotel to our result list.

Let us consider another scenario where the user is looking for hotel for price of less than 300.00 with markup of $20 \%$ in the location Falmouth with some facilities selected such as Swimming pool, Local tours, Bar. Based on the search, database agent could not find hotels for price less than 300.00 in location Falmouth, and found hotel for criteria in neighboring location of 
Montego Bay which are shown in Fig. 13 with rating above 3 and popularity above $60 \%$ which is excellent.

\section{B. Hotel Search with Agent Learning}

Prior to now we have seen how the user selects the criterion which includes price, markup and also facilities. The facilities if left blank the agent would take some default value and return the results. So we here bring the learning capability of search agent where it is enough the user gives the basic criteria for search i.e. Location, Price, Price markup, ratings and other parameters such as Facilities are left as blank. Here the search agent does not search hotels taking the default value for facilities but possess the learning capability to search for hotels for a particular price with facilities which is most commonly been used by users based on popularity information. This shows the past search experience and learning capability of search agent similar to what we would experience with the human travel agent. These will be shown as screenshots for few scenarios

Let us consider a scenario where the user is looking for a hotel in a location Kingston for price of 200.00 with some facilities like Internet Access, Restaurant, Swimming Pool and Activities like Tennis and Local Tours. In here the agent finds no hotel for your price with the facilities asked for in your location and neighboring location. In this situation the agent uses the agent learning to retrieve hotels of any type for price less than 200.00 and hotels of any price matching all facilities asked in location Kingston or in neighboring location based on popularity rather than retrieving hotels with best matched or saying no hotels found to revise the search criteria by the user. Result listing in Fig. 14 here shows two hotels- one meeting the cost requirement and the other however meeting the facilities asked for.(Restaurant, internet, swimming pool, tennis and local tours) hence meeting both requirements above. This is shown in Figs 15 and 16 which has all facilities offered by both hotels. Hence agent intelligence is relentlessly at work for the user trying to find a hotel. Hotels are accurately ranked according to popularity index and ratings shown based on agent intelligence.

Let us consider another scenario where the user is searching for a hotel in a location Kingston for price less than 100.00. Now by applying Agent learning, database agent looks for a hotel for price of $\$ 100.00$ in same or different location and agent with its past search experience is not able to find the hotel in the location Kingston or neighboring location for this price. So the agent here search for hotel with his past search experience and retrieve the hotel/s for any price in location Kingston or neighboring location based on popularity as shown in Fig. 17. By applying agent learning, Agent found all hotels based on popularity in same or neighboring location instead of returning no results found. Once more the agent was able to not only find the hotels but present them ranked by popularity and show relevant ratings associated with each hotel so that the prospective guest can make the best informed decision of where to stay for his/her vacation or business trip.

Let us consider last and final scenario where user selects very minimal criteria for search i.e. location, Price, markup and feedback which is 3 months. In here the user selects the location i.e. Kingston from Google Map. Here the user does not select any facilities towards hotel search. Now based on agent learning capability with the past search experience, the database agent looks for hotels for price 300.00 with markup of $30 \%$ in the location Kingston and retrieves hotel according to popularity as shown in Fig. 18.

\section{Rating and Popularity on Mobile Device}

The rating for the Strawberry Hill Hotel is displayed below in Fig. 19 which displays a rating of three for the Strawberry Hill Hotel and Spa. While a comprehensive listing of all categories are given in Fig. 19 which states exactly how area was scored. The Feedback Agent (FA) is also crucial to the functionality of this section for analysis and presentation. Hotels are issued a warning once the rating falls below three or popularity index is average (15 to 30 percent) or below. After which they are given a maximum of six months to improve on their service before being automatically dropped from the listing for any new search perform by a potential guest. This message will be displayed for mobile users and the hotel administrative staff to see. Once again demonstrating the intelligence implemented on all frontiers of the system as shown in Fig. 20

\section{Administration Popularity and Rating}

The administration interface also provides the facility for viewing of the rating and popularity index of each hotel by region. This facilitates the hotel to view progress and feedback from guests about their hotel chain All hotels that are rated and booked that are located in the selected locations will form part of the data for analysis, interpretation and display for the hotel user This will provide accurate, readable and relevant data for the hotel administrative staff. Listing of all hotels in Kingston (Smart Agent processing) with warning message displayed bottom right for the last item in the list. This list is also ranked by popularity of each hotel in the given location as shown in Fig. 21.

\section{Conclusion \& Future Work}

Living in the fast paced information age along with the speed at which the channels and information is disseminated at times. The volume can be overwhelming to the user who is searching for the prospective hotel for work or vacation. Added to this is 
the trust factor of conducting electronic commerce when some of the entities themselves cannot provide or even are aware of the pros and cons of conducting online transactions. The architecture presented and ensuing system prudently anticipates the needs of the user and makes smart decisions based on fuzzy preference rules that are most desirable for the potential guest. The proposed system also features a security layer to the booking and cancellation process which seeks to protect both the guest and hotel from different levels of potential fraud or misrepresentation that could leave any of the participants involved with some financial loss or other ill-fated forms of inconvenience which has been published elsewhere.. Hotel administrative staffs are allowed via a desktop implementation developed using JADE4.0 and Java jdk1.6_25 to view ratings, popularity index and effect cancellations.

The proposed Smart Agent Learning for Hotel Search System can be improved by developing remainder of check-in dates that can be facilitated via email and or SMS (Short Message Service) medium. This would alleviate some of the inconveniences for the hotel and helpfully aid for the potential guest. When viewing the facilities of the hotel, links to hotels web site could be helpful in terms of giving the guest a more meaning view of the hotel in question as they often have images of the property and in some case clips, which are tours of the property. This would allow a clear impression and give a vivid picture to the potential guest of the hotel. Last but not the least Google maps could be utilized more in providing a street view of the hotel and not just the overhead view of the property and immediate surroundings.

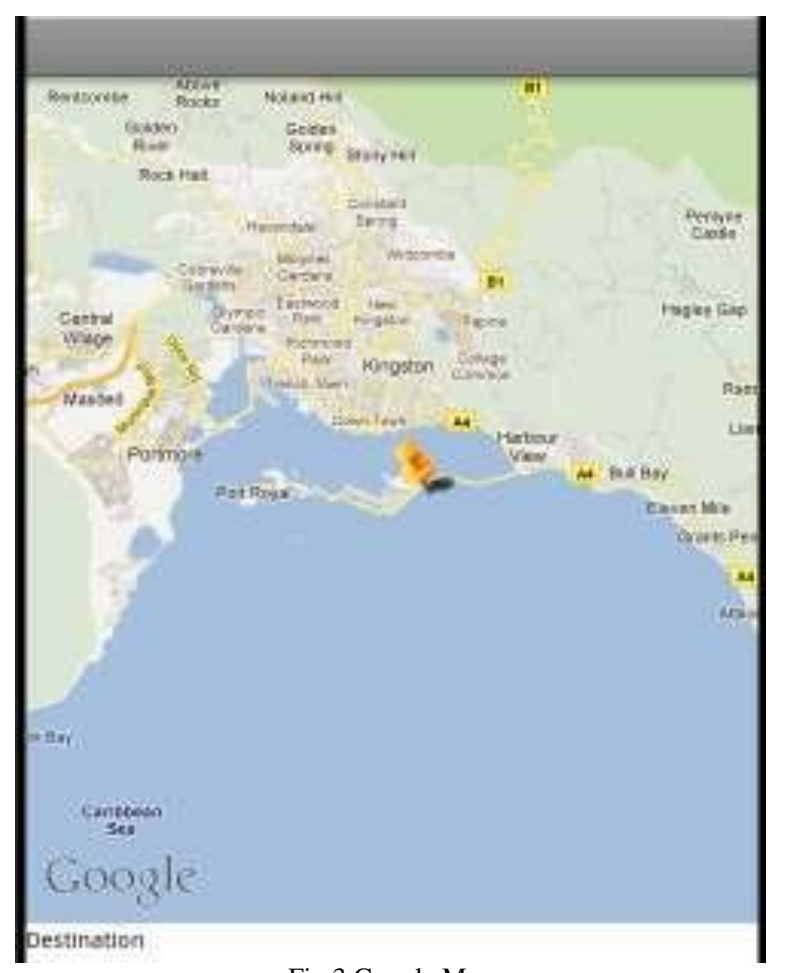

Fig 3 Google Map

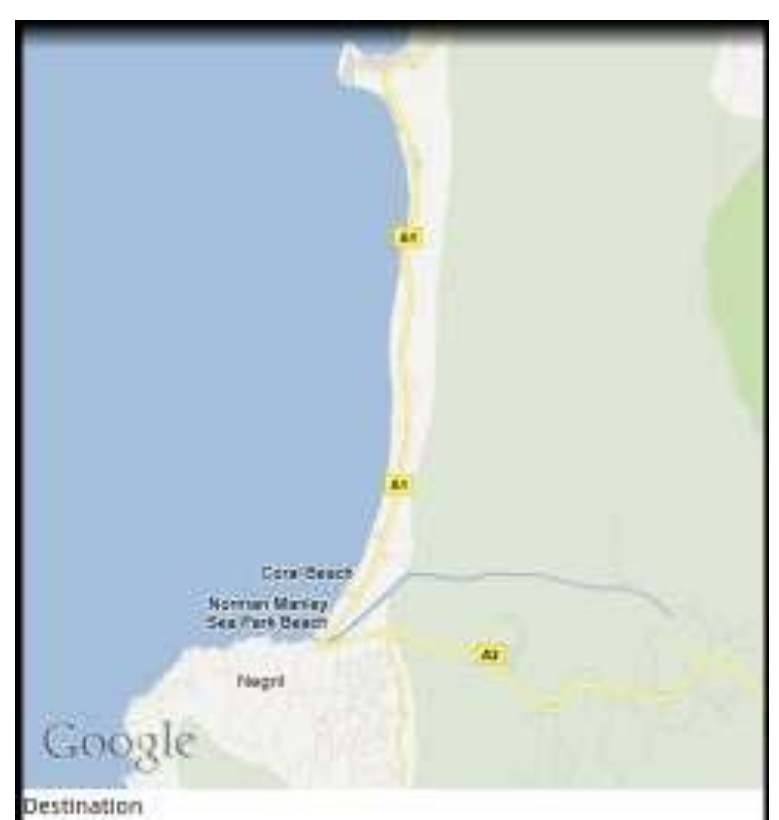

Fig 4 Location Selection

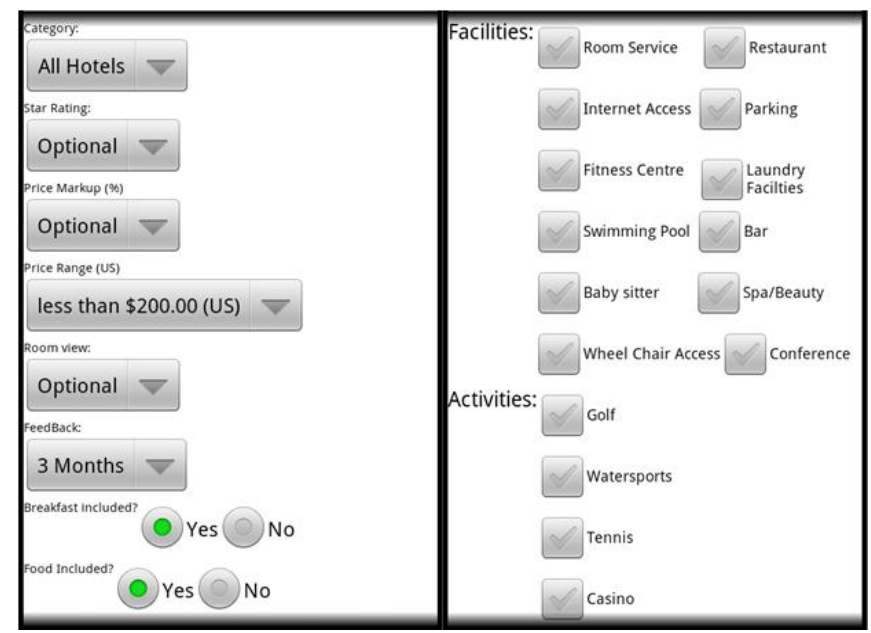

Fig 5 Search Criteria

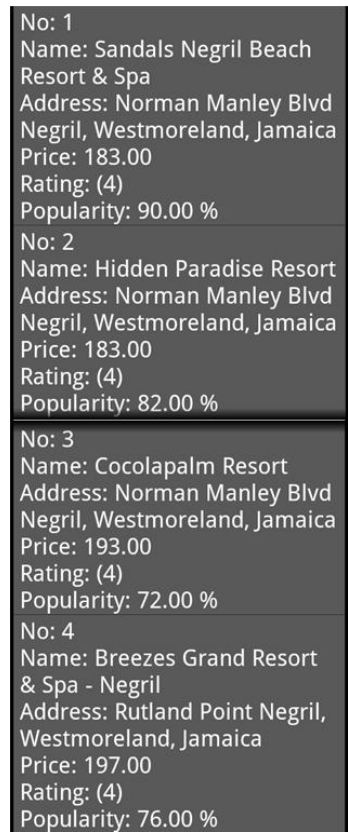

Fig 6 Hotel Search Results-1 


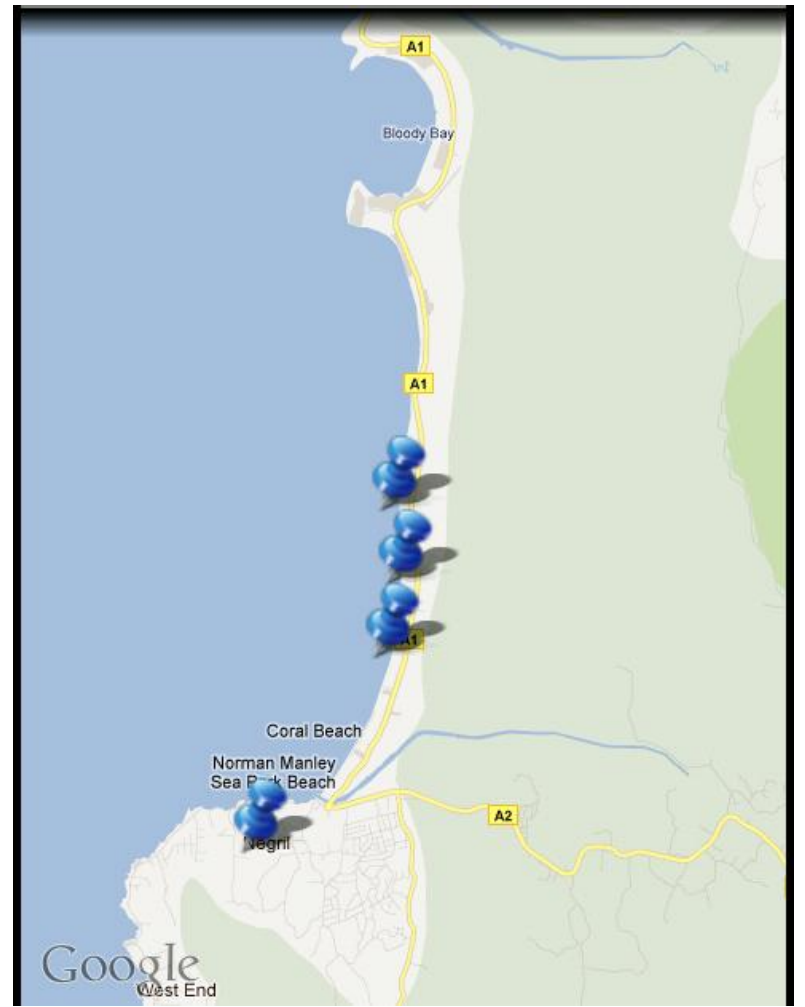

Fig 7 Google Map Hotel Location

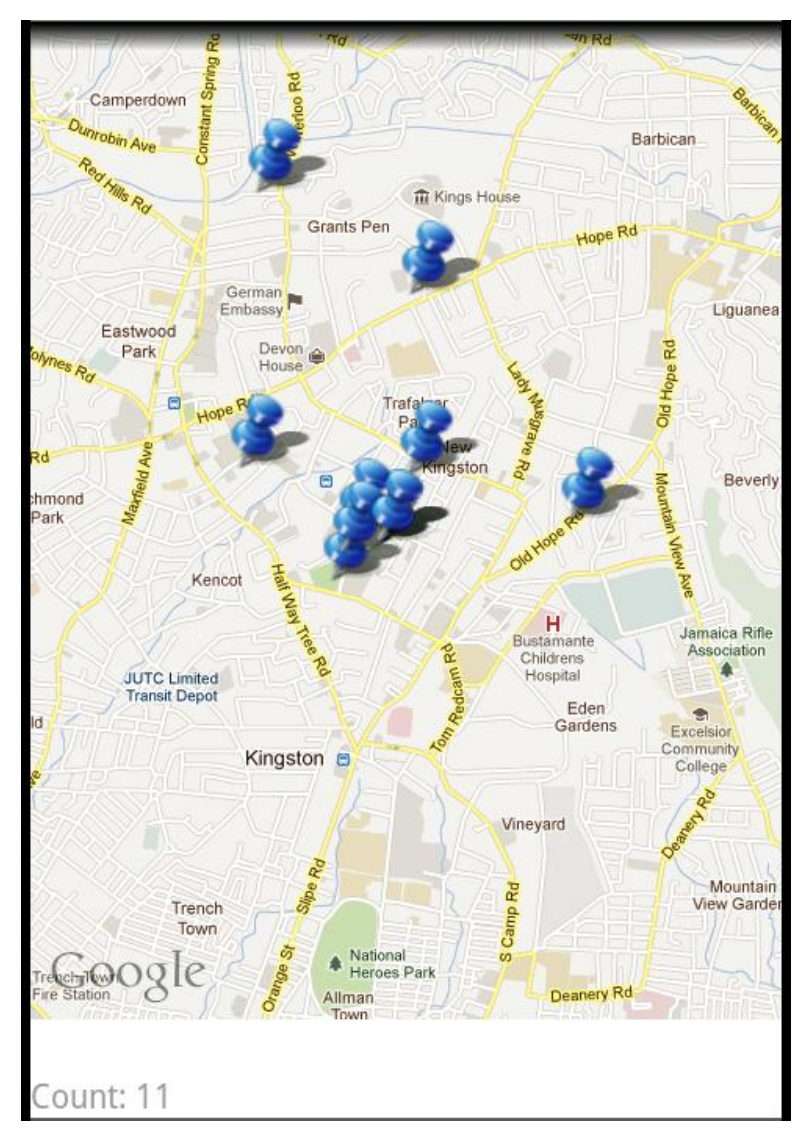

Fig 9 Google Map Hotel Location

\begin{tabular}{l} 
No: 1 \\
Name: Wyndham Kingston \\
Jamaica \\
Address: 77 Knutsford Blvd \\
Kingston 5, Jamaica \\
Price: 159.00 \\
Rating: (0) \\
Popularity: $0.00 \%$ \\
No: 2 \\
Name: Strawberry Hill Hotel \& \\
Spa \\
Address: Irish Town, Saint \\
Andrew, Jamaica \\
Price: 195.00 \\
Rating: (3) \\
\hline No: 3 \\
Name: Jamaica Pegasus Hotel \\
Address: 81 Knutsford Blvd \\
Kingston 5, Jamaica \\
Price: 280.00 \\
Rating: (4) \\
Popularity: $33.33 \%$ \\
No: 4 \\
Name: Courtleigh Hotel \& \\
Suites \\
Address: 85 Knutsford Blvd, \\
Kingston 5, Jamaica \\
Price: 167.00 \\
Rating: (0) \\
Popularity: 0.00 \%
\end{tabular}

Fig 10 Hotel Search Result-3 


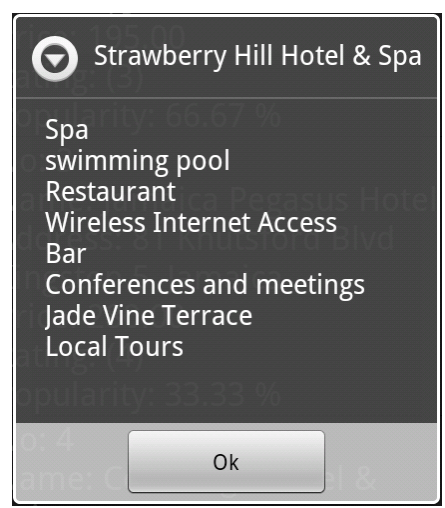

Fig 11 Stawberry Hill Hotel

Jamaica Pegasus Hotel
Facilities:
Business Centre
Conference facilities
Gym
Laundry Service
Parking
Restaurant
Room service
Swimming Pool
Tennis court
Disabled facilities
Internet Access
Cocktail bar
Spa
Cafe
Terrace
Safe
Wheelchair Accessible
Air Condition
Reception area
24h check-in
24h Reception
Currency Exchange
Lift
Shop-s

\begin{tabular}{l}
\hline Shop-s \\
Hairdresser \\
Bar \\
Casino \\
Restaurant - non-smoking area \\
Restaurant -smoking area \\
High chairs \\
Public Internet \\
Wireless Internet access \\
Car park \\
Playground \\
Outdoor swimming pool \\
Children's swimming pool \\
Snack / pool bar \\
Sun loungers \\
Parasols \\
Whirlpool \\
Sauna \\
Sunbathing Terrace \\
Massage \\
Tennis \\
\hline
\end{tabular}

Fig 12 Jamaica Pegasus Facilities

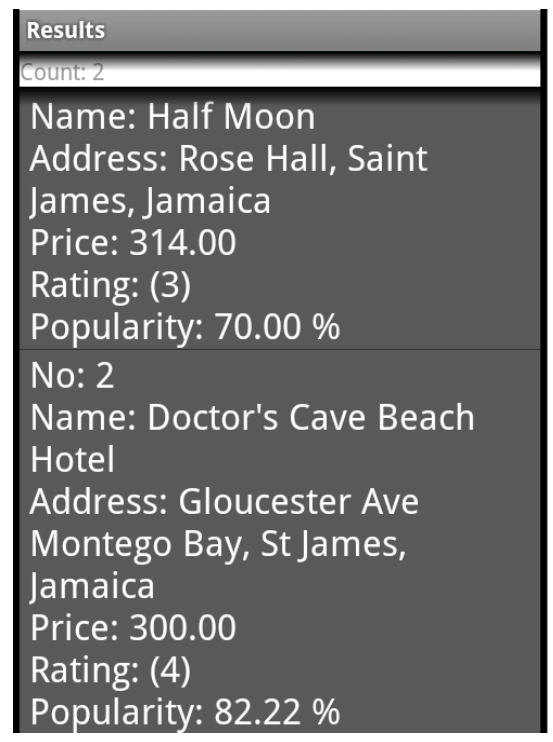

Fig 13 Hotel Search Results-4

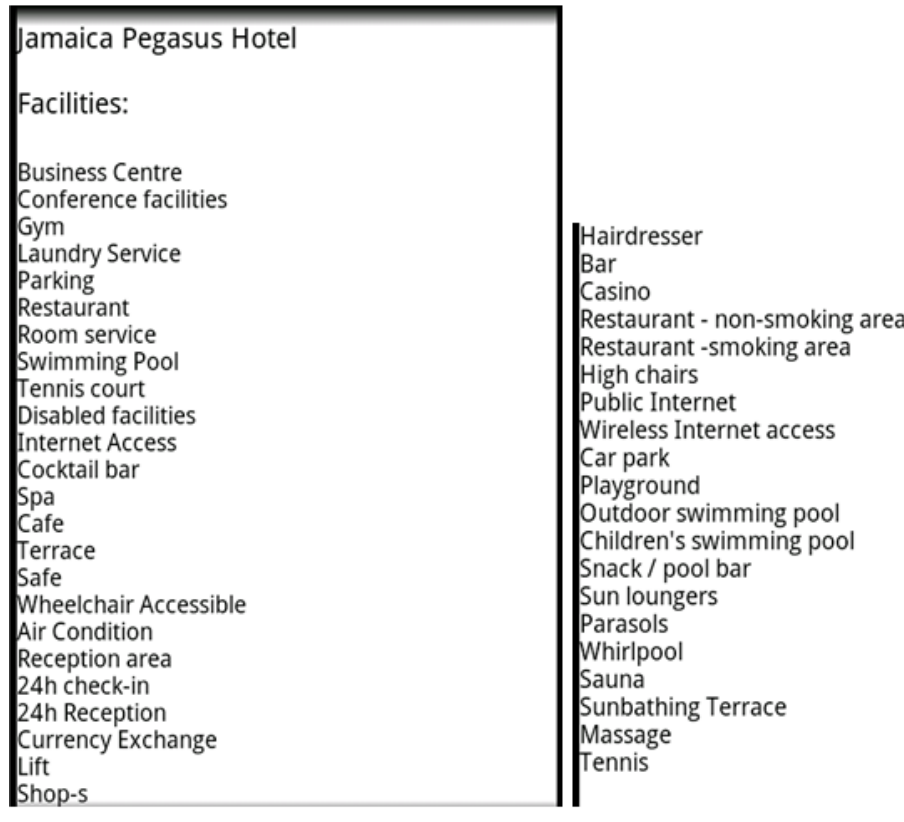

2
No: 1

Name: Strawberry Hill Hotel \& Spa

Address: Irish Town, Saint

Andrew, Jamaica

Price: 195.00

Rating: (3)

Popularity: $66.67 \%$

No: 2

Name: Jamaica Pegasus Hotel

Address: 81 Knutsford Blvd

Kingston 5, Jamaica

Price: 280.00

Rating: (4)

Popularity: $33.33 \%$

Fig 14 Hotel Search Results-5

Strawberry Hill Hotel \& Spa

Spa

swimming pool

Restaurant

Wireless Internet Access

Bar

Conferences and meetings

Jade Vine Terrace

Local Tours

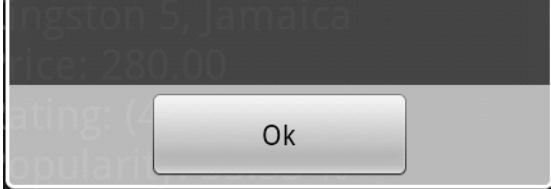

Fig 15 Hotel with Exact Price

Fig 16 Hotel with exact matching of Facilities 


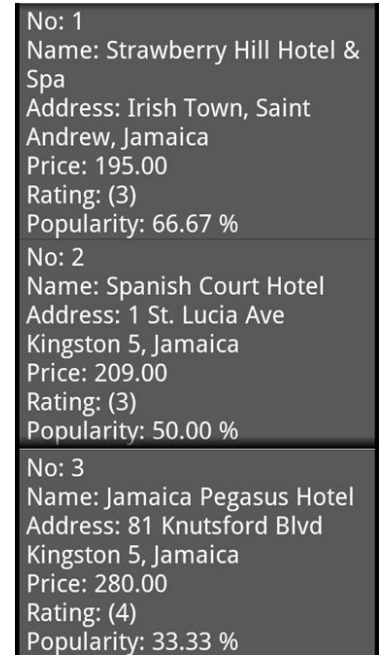

Fig 17 Hotel Search Results-6

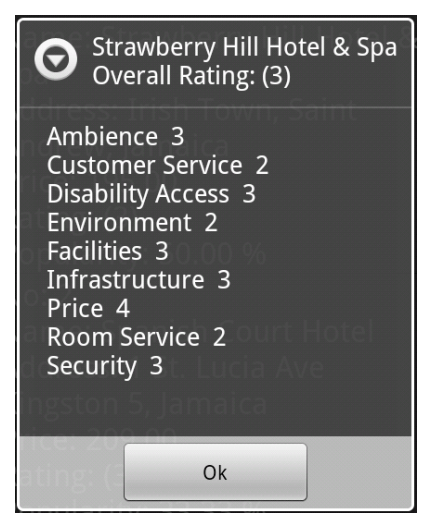

Fig 19 Hotel Rating Details

\begin{tabular}{|c|c|}
\hline $\begin{array}{l}\text { No: } 1 \\
\text { Name: Spanish Court Hotel } \\
\text { Address: } 1 \text { St. Lucia Ave } \\
\text { Kingston 5, Jamaica } \\
\text { Price: } 379.00 \\
\text { Rating: (3) } \\
\text { Popularity: } 75.00 \% \\
\text { No: } 2 \\
\text { Name: Jamaica Pegasus Hotel } \\
\text { Address: } 81 \text { Knutsford Blvd } \\
\text { Kingston 5, Jamaica } \\
\text { Price: } 300.00 \\
\text { Rating: (4) } \\
\text { Popularity: } 33.33 \% \\
\end{array}$ & $\begin{array}{l}\text { No: } 3 \\
\text { Name: Roosevelt Guest House } \\
\text { Address: } 3 \text { Latham Av, } \\
\text { Kingston } 6 \text {, Jamaica } \\
\text { Price: } 380.00 \\
\text { Rating: }(0) \\
\text { Popularity: } 0.00 \% \\
\text { No: } 4 \\
\text { Name: Knutsford Court Hotel } \\
\text { Ltd } \\
\text { Address: } 11 \text { Ruthven Rd } \\
\text { Kingston } 10, \text { Jamaica } \\
\text { Price: } 342.00 \\
\text { Rating: }(0) \\
\text { Dannlariti.. }\end{array}$ \\
\hline $\begin{array}{l}\text { No: } 5 \\
\text { Name: Wyndham Kingston } \\
\text { Jamaica } \\
\text { Address: } 77 \text { Knutsford Blvd } \\
\text { Kingston } 5 \text {, Jamaica } \\
\text { Price: } 359.00 \\
\text { Rating: (0) } \\
\text { Popularity: } 0.00 \% \\
\text { No: } 6 \\
\text { Name: Terra Nova All-Suite } \\
\text { Hotel } \\
\text { Address: } 17 \text { Waterloo Rd, } \\
\text { Kingston } 10 \text {, Jamaica } \\
\text { Price: } 355.00\end{array}$ & $\begin{array}{l}\text { No: } 7 \\
\text { Name: Sunset Inn Hotel } \\
\text { Address: 1A Altamont Cres } \\
\text { Kingston 5, Jamaica } \\
\text { Price: } 375.00 \\
\text { Rating: (0) } \\
\text { Popularity: } 0.00 \% \\
\text { No: } 8 \\
\text { Name: Starlight Chalet \& } \\
\text { Health Spa } \\
\text { Address: } 21 \text { Rochester Ave, } \\
\text { Kingston 8, Jamaica } \\
\text { Price: } 386.00 \\
\text { Rating: (0) }\end{array}$ \\
\hline
\end{tabular}

Fig 18 Hotel Search Results-7

No: 11
Name: Abahati Hotel
Address: 7 Grosvenor Terrace
Kingston 8, Jamaica
Price: 150.00
Rating: (1)
Popularity: $0.00 \%$
Notice:Hotel has bad review/s.
improve service within 3 to 6
month's time.

Fig 20 Warning Message

\begin{tabular}{|c|c|c|c|c|c|c|}
\hline \multicolumn{3}{|l|}{ Ratings \& Popularity } & & & \multicolumn{2}{|c|}{\begin{tabular}{|l|l|l|}
0 & ⿴囗口 & $x$ \\
\end{tabular}} \\
\hline Name & Address & Cost & Rating & Popularity & Notice & \\
\hline Spanish Court Hotel & 1 St. Lucia Ave Kingston 5, Ja... & 209.00 & 3 & 50.00 & & A \\
\hline Jamaica Pegasus Hotel & 81 Knutsford Blvd Kingston $5, \ldots$ & 280.00 & 4 & 33.33 & & \\
\hline Wyndham Kingston Jamaica & 77 Knutsford Blvd Kingston $5, \ldots$ & 159.00 & 0 & 0.00 & & \\
\hline Terra Nova All-Suite Hotel & 17 Waterloo Rd, Kingston $10, \ldots$ & 613.00 & 0 & 0.00 & & \\
\hline Sunset Inn Hotel & 1A Altamont Cres Kingston $5, \ldots$ & 375.00 & 0 & 0.00 & & \\
\hline Stony Hill Spa Hotel & Hermitage Dam Rd Stony Hill, .... & 651.00 & 0 & 0.00 & & \\
\hline Starlight Chalet \& Health Spa & 21 Rochester Ave, Kingston $8 \ldots$ & 386.00 & 0 & 0.00 & & \\
\hline Springburn House & 1 Springburn Av, Kingston $19, \ldots$ & 549.00 & 0 & 0.00 & & \\
\hline Shirley Retreat Hotel & 7 Maeven Av Kingston 10, Ja... & 808.00 & 0 & 0.00 & & $\equiv$ \\
\hline Roosevelt Guest House The & 3 Latham Av, Kingston 6, Jam... & 380.00 & 0 & 0.00 & & \\
\hline Robin's Bay Village \& Beach R... & 15 Red Hills Rd Kingston $10, \mathrm{~J} . .$. & 558.00 & 0 & 0.00 & & \\
\hline Prestige The & 70 Sandhurst Cres Kingston $6 \ldots$ & 251.00 & 0 & 0.00 & & \\
\hline Paradise Resorts Mgmt & 21 Hialeah Dr, Kingston 19, Ja... & 638.00 & 0 & 0.00 & & \\
\hline Olympia Crown Hotel & 53 Molynes Road, Kingston $10 . .$. & 391.00 & 0 & 0.00 & & \\
\hline Morgan's Harbour Hotel & Port Royal, Kingston 1, Jamaica & 650.00 & 0 & 0.00 & & \\
\hline Mona Visitor's Lodge \& Confer... & Mona, Kingston 7, Jamaica & 505.00 & 0 & 0.00 & & \\
\hline Medallion Hall Hotel & 53 Hope Rd Kingston 6, Jamaica & 497.00 & 0 & 0.00 & & \\
\hline Mayfair Hotel & 4 West Kings Hse Cl Kingston ... & 623.00 & 0 & 0.00 & & \\
\hline Knutsford Court Hotel Ltd & 11 Ruthven Rd Kingston $10, \mathrm{~J} . .$. & 342.00 & 0 & 0.00 & & \\
\hline Jartena Apts Ltd & 1 Altamont Terrace, Kingston ... & 458.00 & 0 & 0.00 & & \\
\hline Island Outpost & 4 Hopefield Ave Kingston $10, \ldots$ & 535.00 & 0 & 0.00 & & \\
\hline Indies Hotel & 5 Holborn Rd Kingston 10, Ja... & 279.00 & 0 & 0.00 & & \\
\hline Hotel Four Seasons & 18 Ruthven Road, Kingston $1 . .$. & 785.00 & 0 & 0.00 & & \\
\hline Goblin Hill Villas At San San & 11 East Av, Kingston 10 , Jam... & 656.00 & 0 & 0.00 & & \\
\hline Gardens & 23 Liguanea Ave, Kingston $6, \ldots$ & 485.00 & 0 & 0.00 & & \\
\hline Eden Gardens & Suite 1839 Lady Musgrave Ro... & 201.00 & 0 & 0.00 & & \\
\hline Crieffe Court Guest House & 10 Crieffe Rd Kingston 6, Jam... & 184.00 & 0 & 0.00 & & \\
\hline Courtleigh Hotel \& Suites & 85 Knutsford Blvd, Kingston 5... & 167.00 & 0 & 0.00 & & \\
\hline City View & Lot 3 Bellevue Smokey Vale Ki... & 491.00 & 0 & 0.00 & & \\
\hline Christar Villas Hotel & 99A Hope Road, Kingston 6, J... & 337.00 & 0 & 0.00 & & \\
\hline Chelsea Hotel & 5 Chelsea Ave, Kingston $10, \mathrm{~J} . .$. & 483.00 & 0 & 0.00 & & \\
\hline Caribbean Villa & Lot 7 St Thomas Rd Kingston ... & 690.00 & 0 & 0.00 & & \\
\hline Brown Barrington & 7 Beechwood Ave Kingston $5, \ldots$ & 531.00 & 0 & 0.00 & & \\
\hline Breezes Resorts \& Spa & 2 St Lucia Ave Kingston 5, Ja... & 177.00 & 0 & 0.00 & & \\
\hline Altamont Court Hotel & 1 Altamont Terrace, Kingston ... & 340.00 & 0 & 0.00 & & \\
\hline Abahati Hotel & 7 Grosvenor Terrace Kingston... & 150.00 & 1 & 0.00 & Hotel has bad review/s. improve service within 3 to 6 month's time. & - \\
\hline
\end{tabular}

Fig 21 Rating and popularity Listing 


\section{References}

[1] Baleleng, T (2011). Hotel Reservation Techniques Retrieved from http://artificialintelligenceseo.info/2011/09/hotelreservation-techniques

[2] Baloch, H (1999). Online Intelligent Hotel Reservation (IHR) using WAP Enable Mobile Phones. Retrieved from http://www.pardis.ir/articles/pdf/p06139.pdf

[3] SSLGUARD (2010). Server-Gated Cryptography Retrieved from http://www.sslguard.com/datasheets/Thawtesgc.pdf

[4] Bailey, L S and Suresh, S(2009). Intelligent Agent based Mobile Tour Planner. Proceedings of First Caribbean Conference on Information and Communication Technology, Kingston, Jamaica. Pp 8-13.

[5] McTavish, C A and Suresh, S (2010). Intelligent Agent based Hotel Search \& Booking System. Proceedings of 2010 IEEE International conference on Electro/Information Technology, Chicago, USA, May 20-22, 2010.pp.331-336

[6] Lawrence, W (2011). Application of Agents in Hotel Search and Secured Booking System. M.sc computer Science Dissertation, Department of Computing, University of WestIndies, Jamaica

[7] Lawrence, W and Suresh, S(2012). "Application of Biometric Security in Agent based Hotel Booking System- Android Environment", Communicated to International Journal on Cryptography and Information Security, AIRCC publishers

[8] Ding et al, (2003), “On Programming Information Agent Systems - An Integrated Hotel Reservation Service as Case Study". Proceedings of First German Conference on Multiagent System Technologies, MATES 2003, Erfurt, Germany.

[9] Gobin, B A and Subramanian R K (2007). Knowledge Modeling for a Hotel Recommendation System, World Academy of Science, Engineering and Technology, Vol.25. pp 244-248.

[10] Franklin, S. and Graesser, A. (1996). Is it an Agent, or just a Program? A Taxonomy for Autonomous Agents. Retrieved from http://www.msci.memphis.edu/ franklin/AgentPro g.html

[11] Jennings, N. R., and Wooldridge, M. (1998). Applications of Intelligent Agents. Retrieved from http://agents.umbc.edu/introduction/jennings98.pd f

[12] Poole et al. (1998). Computational Intelligence. New York: Oxford University
[13] Reilly, D. (1998). Mobile Agents - Process migration and its implications. Retrieved from http://www.davidreilly.com/topics/software_agent s/mobile-agents

[14] Serenko, A. and Detlor, B. (2002). A General Overview of the Market and an Assessment of Instructor Satisfaction with Utilizing Toolkits in the Classroom. Retrieved from Agent Toolkits: http://foba.lakeheadu.ca/serenko/Agent Toolkits Working Paper.pdf

[15] Panait, L and Luke, S (2005). Cooperative MultiAgent Learning: The State of the Art Retrieved from

http://cs.gmu.edu/ eclab/papers/panait05cooperati ve.pdf

[16] Bellifemine, F et al (2006). Developing MultiAgen Systems with JADE. Wiley Publishing.

[17] Bellifemine, F et al (2007). JADE: A software framework for developing multi-agent applications. Retrieved from http://www.dia.fi.upm.es/ phernan/AgentesIntelig entes/referencias/bellifemine07.pdf

[18] Google (2011). What is Android. Retrieved from http://code.google.com/android/what-isandroid.html

[19] Microsoft (2007). Microsoft SQL Server 2008 Top New Features. Retrieved from www.microsoft.com/sqlserver/en/us/editions.aspx

Wayne Lawrence is a final year Msc. Computer Science student in the Department of Computing at the University of the West Indies, Jamaica since 2009. Prior to that, he obtained Bachelor's degree in Computing \& Information Technology from University of Technology Jamaica in 2004.

He is currently working as Database Administration in Epayment Group Limited. In his Master's Programme he did Master's thesis on "Application of Intelligent Agents in Hotel Search and Secured Booking System which focused on using intelligent agents to search, the hotels based on user requirements and also booking the hotels online securely suing biometrics. His research interest includes Intelligent Agents, Mobile commerce, Software Engineering and Database.

Prof. Suresh Sankaranarayanan holds a PhD degree (2006) in Electrical Engineering with specialization in Networking from the University of South Australia. He is a Senior Member of IEEE computer Society and Computer Society of India too. He was working as a Lecturer (Asst. Prof. Status) in the Department of Computing and lead the Intelligent Networking Research Group, in the University of West Indies, 
Kingston, Jamaica, during 2008-11.He has also worked as a Professor, School of Computer Science and Engineering, Vellore Institute of Technology (VIT University), Chennai Campus, India, for a short period during 2011. He is now working as Associate Professor, Department of Computer \& Information Systems, Institute of Technology, Brunei (ITB - A technological university). Currently he is also functioning as a Visiting Professor, Department of computing, Faculty of Pure \& applied Science, University of West Indies, Mona Campus, Kingston-7, Jamaica, West Indies. He has got to his credit, about 50 fully refereed research papers published in the Proceedings of major IEEE international conferences, as Book Chapters and in International Journals. $\mathrm{He}$ is also a Reviewer and Technical Committee member for a number of IEEE Conferences and Journals. His current research interests are mainly towards Mobile and Ubiquitous ComputingWireless Sensor Networks in Health \& Engineering, Intelligent Agents, Cloud Computing, Mobile commerce. 